\title{
Desarrollo regional y acción de base: El caso de una organización indígena de productores de café en Oaxaca
}

\section{Regional Development and Grassroots Action: The case of an indigenous organization of coffee producers in Oaxaca}

\author{
Carlos Chávez-Becker* \\ Alejandro Natal ${ }^{* *}$
}

\begin{abstract}
This document answers the question on which factors can make a grassroots organization, composed by poor, ill-educated peasants, marginalized from markets and governments, successful not only in integrating themselves to global markets, but also capable of fostering significant development processes at regional level. We argue that success can only be achieved when three intermingled factors are present: 1) the relations the organization builds with the exterior, its learning from brokerage, and the construction of its own capabilities; 2) internal government mechanisms that, within the organization, foster participation in decision making, representation, plurality, and appropriation among others; and 3) a broad interest in the territory and its needs, what gives the organization the necessary legitimacy to promote collective action for social change and development.
\end{abstract}

Keywords: grassroots action, bottom-up development, cooperatives in rural Mexico, Fair Trade producer's organizations.

\section{Resumen}

Este documento responde a la pregunta, ¿qué factores hacen que una organización de base, compuesta por campesinos pobres, con bajo nivel educativo, marginados por los mercados y el gobierno, sean exitosos al insertarse en los mercados globales y promover procesos de desarrollo en su región? Nuestro argumento es que esto es posible cuando se combinan tres factores: 1) contactos con el exterior que le permiten a la organización construir puentes, aprender y desarrollar capacidades; 2) mecanismos internos de gobierno que posibilitan la participación en la toma de decisiones, así como representación, pluralidad y apropiación, entre otras, y 3 ) un interés amplio en el territorio y sus necesidades por parte de la organización, lo que le gana legitimidad para promover cambio social y desarrollo.

Palabras clave: acción de base, desarrollo de arriba hacia abajo, cooperativas rurales en México, organizaciones de productores campesinos, comercio justo.

* Universidad Nacional Autónoma de México, México. Correo-e: mejalmamut@yahoo.com.

** El Colegio Mexiquense, A.C., México. Correo-e: anatal@gmail.com. 


\section{Introducción ${ }^{1}$}

Con este trabajo buscamos responder la siguiente pregunta: ¿qué factores pueden hacer que una organización comunitaria de base (осB, en inglés grassroot organization) sea exitosa en la promoción del desarrollo regional? Para el caso analizado se muestra que hay tres elementos que se retroalimentan entre sí: las relaciones que una organización construye al exterior, estructuras de gobierno participativas, y la atención al territorio y sus necesidades. El caso de estudio analiza una organización comunitaria vinculada al comercio justo, en Oaxaca, México.

Según diferentes autores (Bauman, 2001; Flores y Mariña, 1999; Ianni 1996), entre muchos otros), entre las externalidades negativas generadas por la globalización está el creciente aislamiento de numerosos segmentos poblacionales. Éste es el caso de campesinos pobres en países en desarrollo, quienes quedan fuera de las nuevas oportunidades del mercado al no ser competitivos ni tener la capacidad para rearticular sus procesos de producción o integrarse a cadenas productivas. Estos grupos, en general, son atendidos por políticas públicas ineficientes o quedan al margen de ellas (Rubio, 1996). Más aún, incluso cuando algunos de ellos logran ubicarse en algún eslabón de la cadena productiva o comercial, quedan expuestos a mercados altamente competitivos, complejos y volátiles para los que carecen de instrumentos de navegación. De tal suerte, en muchos casos se genera una espiral descendente que dificulta que los productores inicien procesos de desarrollo propios que les permitan salir de la pobreza y la marginación.

Sin embargo, ante este desalentador escenario, han ido surgiendo formas alternativas mediante las cuales algunos grupos se logran abrir un lugar y sortear las fallas del mercado y del Estado. Una de ellas es el comercio justo (CJ). Paulatinamente este movimiento ha ido ganando reconocimiento como una alternativa económica y sustentable, en especial para pequeños productores no competitivos.

El CJ se basa en dar un precio justo a los productores primarios mediante la disminución de intermediarios entre los productores del Sur y los consumidores del Norte, a fin de aumentar las ganancias de los primeros. ${ }^{2}$ Esto es posible gracias a que el CJ construye cadenas alternativas de comercialización, que trabajan con la filosofía Comercio, no ayuda

${ }^{1}$ Una versión anterior de este documento se presentó en el Congreso New Latin American Development Strategies in a Changing International Economic and Political Context, en la Universidad de Aalborg, Dinamarca, en mayo de 2008.

${ }^{2}$ Oxfam (2002: 24-25) investigó sobre la estructura de precios de un kilo de café producido en Uganda y vendido en el Reino Unido. El estudio concluye que entre el empaquetador, el importador y el distribuidor minorista se queda cerca de $80 \%$ del precio final, que pasa de 14 centavos de dólar que recibe el productor primario, a 26.40 dólares cuando lo adquiere el consumidor. 
(Trade not aid) (Fridell, 2006), y se abocan a reducir costos de transacción y barreras de entrada que enfrentan los productores para tener acceso a mercados globales. Después de tres décadas este movimiento ha crecido de forma notable, al punto que hoy genera recursos para más de un millón y medio de productores pobres (unos 7.5 millones de personas) y sus organizaciones de base (827) en aproximadamente 60 países en desarrollo (FLO, 2010: 14); alcanzando ventas récord de 3.4 billones de euros ${ }^{3}$ en el último ejercicio. ${ }^{4}$

En este documento exploramos cómo una осв vinculada al CJ ha detonado procesos de desarrollo regional y acción de base, siendo muy exitosa en utilizar alianzas y estructuras organizativas locales. Esta осв es la Unión de Comunidades Indígenas de la Región del Istmo (UCIRI), una organización indígena-campesina pionera del movimiento de cJ, y una de las primeras en eliminar a parte de los intermediarios y en exportar desde el Sur a países desarrollados (Jaffee, 2007; Fridell, 2007; Aranda y Morales, 2002; Simpson y Rapone, 2000). La UCIRI ha jugado un papel de liderazgo al interior del movimiento del cJ, contribuyendo enormemente a su fortalecimiento y expansión en el mundo (Simpson y Rapone, 2000). Pero lo más importante para los efectos de este trabajo, es que esta organización se ha convertido también en un catalizador de procesos de desarrollo local en la región a la que sirve, mostrándonos con ello las posibilidades que puede tener una organización comunitaria, rural e indígena en la distribución de la riqueza y como detonadora de procesos de desarrollo local.

La Unión $n^{5}$ nace en $1983^{6}$ como respuesta desde las bases a: 1) la paulatina depreciación del precio internacional del café; ${ }^{7}$ 2) a un mercado controlado por intermediarios o coyotes y acaparadores; 3) falta de créditos, y 4) al escaso apoyo recibido por parte del Instituto Mexicano del Café (Inmecafé). Todos estos factores deterioraban sensiblemente las

${ }^{3}$ Datos para 2009 según flo (2010: 12).

${ }^{4}$ No obstante, a pesar de su notable crecimiento y de su potencial, el cJ no ha tenido la suficiente atención académica que nos permita aprender las lecciones que este movimiento tiene para el desarrollo, y en particular para la detonación de procesos de cambio social en las comunidades de productores pobres. A la fecha, la mayoría de los trabajos se han enfocado a evidenciar su efecto en el ingreso de los productores (Pariente, 2000; Jolette, 2001; Ronchi, 2002; Reynolds et al., 2003; Durnez, 2004; Utting, 2005), o al análisis de las relaciones entre ong del Norte y del Sur, o de redes internacionales. De tal suerte, a la fecha hay aún un número de áreas que necesitan atención, como la sustentabilidad de las organizaciones locales, su efecto en su entorno económico y social, su legitimidad al interior de las comunidades, entre otras.

${ }^{5}$ Usamos los términos de la Unión, la organización o la UCIRI, indistintamente.

${ }^{6}$ En 1981-1982, con la asesoría de la Diócesis de Tehuantepec (en especial de Frans Vanderhoff), se vendió parte de la cosecha a través de la Asociación Rural de Interés Colectivo (ARIC) de Misantla, Veracruz, recibiendo un pago de 95 centavos de dólar, mayor al pagado por los coyotes de Ixtepec ( 25 centavos).

${ }^{7}$ Desregulado a partir de 1989 con el colapso del Acuerdo Internacional del Café, que limitaba las cuotas de venta y exportación de países productores. 
condiciones de vida de los productores y, en muchos casos, no les dejaban más opción que la migración. Por ello, la creación de la UCIRI significó una esperanza para mejorar las condiciones de comercialización.

Durante los primeros años la UCIRI, a través del tequio, desarrolló su propia infraestructura y se fue apropiando de algunos eslabones del proceso de comercialización, hasta obtener en 1985 la licencia de exportación (Simpson y Rapone, 2000: 50). Paralelamente, la Unión realizó un esfuerzo constante para mejorar los procesos productivos, ańadiendo así valor agregado a su producto.

Estos logros fueron interesando a otros productores de la región, y al poco tiempo de su creación la organización registraba un intenso crecimiento en membrecía, alcance territorial y capacidad productiva. Esta tendencia continuaría durante tres décadas, y para el año 2010 la UCIRI ya sería una organización con 2,400 socios, todos ellos indígenas (de las etnias mixteca, chontal, mixe, chatina y zapoteca) provenientes de 58 comunidades de 20 municipios del norte y centro de Oaxaca. Por otro lado, la Unión también registró un importante crecimiento en su capacidad de acopio y producción de café, ya que de producir unas cuantas toneladas, a principios de los ochenta, vendió casi mil toneladas en 2010. Pero además de sus éxitos de comercialización, la Unión comenzó a jugar un papel importante como una organización representativa de las comunidades de la región, como integradora de grupos étnicos y generadora de consensos. De tal suerte que pronto se involucró en proyectos que resolvían las necesidades apremiantes de la población de la zona, como una cooperativa de transporte, tiendas comunitarias, una ferretería, escuelas, clínicas de salud y talleres; amén de importantes procesos sociales que potenciaron notablemente el empoderamiento y la autogestión. Así las cosas, a la vuelta de 30 ańos la Unión es un verdadero polo de desarrollo en una región caracterizada por el conflicto, la pobreza y la marginación (Jaffee, 2007: 37-40; Aranda, 2003: 156; Fridell, 2007: 175).

Por tanto, la pregunta que surge es, ¿cómo una organización indígena, conformada por pequeños y pobres productores primarios en el México rural, ha logrado vencer los enormes retos que representa integrarse al mercado global y detonar el desarrollo regional en contextos políticos y económicos adversos?

En las siguientes secciones analizamos con más detalle los tres factores que consideramos clave para responder esta pregunta. El primero, la capacidad de la organización para establecer alianzas con diversos actores, aprovecharlos para tender puentes, o brokerage, y desarrollar capacidades propias a partir de esta relación. El segundo factor, la capacidad de la organización para construir un gobierno interno que le diera legitimidad y apropiación vis-a-vis y de parte de sus miembros. El tercero, la capacidad 
de la organización para traducir estos vínculos con el exterior y solidez interna en procesos locales de cambio social, o sea, su aptitud para modificar en beneficio de sus miembros su entorno inmediato. Entendemos que estos tres procesos son simultáneos y dialécticos, pero consideramos que para efectos analíticos pueden ser diferenciables.

Para llevar a cabo el análisis de estos factores dividimos el presente trabajo en cuatro partes. Primero presentamos cómo la organización construyó alianzas en el exterior que le permitieron ir adquiriendo capacidades y empoderarse. Segundo, mostramos cómo ciertos mecanismos de gobierno le han dotado a la organización de una gran legitimidad y cómo, dichos mecanismos, han fortalecido un proceso de apropiación de la organización entre sus agremiados. Tercero, analizamos cómo con la experiencia adquirida y siendo una organización sólida, la UCIRI se convirtió en un polo de desarrollo regional. Terminamos con un breve apartado de conclusiones que pueden servir como punto de partida para una, muy necesaria, discusión sobre el papel de las осв en el desarrollo regional.

Cabe mencionar que para la elaboración de este trabajo se utilizó información recopilada en entrevistas a profundidad y observación participante en diversas comunidades en las que la UCIRI tiene membrecía. Las entrevistas se realizaron a socios productores de la organización y a otro tipo de informantes clave, como ex socios de la organización, miembros de otras organizaciones y asesores de la Unión. El criterio central que rigió la selección de entrevistados fue una división entre socios antiguos, preferentemente fundadores de la organización, y socios de reciente ingreso, con el objetivo de contrastar sus puntos de vista sobre aspectos tan importantes como la legitimidad interna de la organización o la confianza en los líderes y autoridades, entre otros aspectos. Las entrevistas que se hicieron a los socios productores fueron a partir de un guión general. Las entrevistas a los informantes clave se hicieron sin un guión de esta naturaleza debido a que cada uno de ellos podía aportar información relevante en temas diversos.

\section{Las alianzas con el exterior}

A partir de la década de los ochenta, la UCIRI buscó y recibió apoyo con algunas organizaciones del movimiento de CJ como las Organizaciones de Comercio Alternativo (ocA), Gesellshaft und Partnerschaft (Alemania), la firma Hans Levelt (Holanda), y Naturland, que otorgó el primer certificado orgánico al café de la UCIRI. En 1987 la Unión colaboró con la 
agencia ecuménica de desarrollo Solidaridad ${ }^{8}$ para crear el sello holandés Max Havelaar, materializando el primer esquema de cJ con certificación auditada (Arditi y Chávez, 2006). ${ }^{9}$ En 1989 la UCIRI es la primera organización en recibir el certificado de producción orgánica, hasta entonces destinado a productores individuales. Paralelamente, la UCIRI se integró a la International Federation of Organic Agriculture Movements (Federación Internacional de Movimientos de Agricultura Orgánica, IFOAM) y formó parte del consejo directivo del sello Max Havelaar (de 1989 a 1992). Posteriormente, en 1997, la UCIRI participa activamente en la creación de Fair Trade Labelling Organizations International (FLO), organización que aglutina a los más de 20 sellos de certificación de CJ y le ha dado al movimiento una estructura más coherente y profesional.

En materia comercial, la UCIRI también creó importantes redes de comercialización que le permitieron explotar el potencial de sus productos certificados. Así, en 1990 comenzó a exportar café a Estados Unidos con la organización Equal Exchange. A lo largo de esa década y los primeros años de la de 2000, la UCIRI amplía también su base de clientes vendiendo a empresas en Europa, Japón, Canadá y Estados Unidos; y en 2004 establece un acuerdo con las empresas francesas Malongo y Carrefour para vender una significativa parte de su producción. En buena medida las negociaciones con estas empresas se realizaron sin la intermediación de organismos internacionales que hasta entonces habían apoyado a la UCIRI.

Otro espacio en el que la UCIRI crea alianzas importantes es con sus pares. Entre las organizaciones de productores de café nacionales, la Unión ganó un indudable liderazgo por su capacidad de innovación y por su apertura para compartir métodos, estrategias y contactos. Así, a partir de los ochenta, la organización comenzó a dar apoyo y asesorías técnicas y logísticas a organizaciones de pequeños productores independientes, como la Unión Majomut, Redcafés, la Tosepan Titataniske, e Indígenas de la Sierra Madre de Motozintla San Isidro Labrador (ISMAM). Pronto estas asesorías se hicieron extensivas a organizaciones guatemaltecas, nicaragüenses y hondureñas. En 1989 formó, junto con otras organizaciones, la Coordinadora Nacional de Organizaciones Cafetaleras (CNOC) y la Coordinadora Estatal de Productores de Café de Oaxaca (cepco).

De la misma manera que hizo en el plano internacional, en el ámbito nacional la Unión participó y contribuyó a crear organizaciones encaminadas a mejorar, simplificar y/o abaratar los procesos de certificación y de producción, como la Asociación Mexicana de Inspectores Orgánicos

\footnotetext{
${ }^{8}$ Solidaridad (2006) es una ONG que promueve la justicia económica, política y cultural en los países en desarrollo.

${ }^{9}$ Estos sellos garantizan un precio justo al productor (Arditi y Chávez, 2006: 62) y que los importadores son los únicos intermediarios (Renard, 2005).
} 
(1993) y Ecomex (certificación ecológica, 1994). Por su parte, en 1997 participó en la creación de la Certificadora Mexicana de Productos y Procesos Ecológicos (Certimex) y más adelante apoyó la conformación del sello mexicano Comercio Justo México (2001). De la misma forma, la organización jugó un rol central en la creación de Agromercados (2000) y del Consejo Mexicano de Cafeticultura Sustentable, A.C., la última de las redes de CJ en México. ${ }^{10}$ Más recientemente, la UCIRI contribuyó a formar la Coordinadora Latinoamericana y del Caribe de Pequeños Productores de Comercio Justo (CLAC). ${ }^{11}$

Los párrafos anteriores muestran que, en primer lugar, las alianzas construidas ayudaron a la UCIRI a tender puentes, o brokerage, para aprovechar oportunidades de comercialización para sus productos en un entorno económico adverso. En segundo, se muestra cómo estas alianzas le permitieron adquirir capacidades y expertise necesaria para moverse en el complejo mundo del comercio internacional, resolviendo por sí misma barreras de entrada y limitaciones que diversos costos de transacción imponían a sus procesos de comercialización. Más aún, y en tercer lugar, la UCIRI ha servido a su vez como puente, o broker, a otros actores societales, convirtiéndose así en un agente (Fama y Jensen, 1963; Fama, 1980) capaz de construir arreglos institucionales para facilitar el CJ y apoyar a otras organizaciones que le podrían significar competencia potencial. Con esto la UCIRI es una fuente de conocimientos e información a la que otras organizaciones pares pueden acceder, convirtiéndose con ello en capital social puente en la región.

De esta manera, la notable evolución de la UCIRI de organización receptora a organización promotora, puede ayudar a entender que los vínculos hacia el exterior son útiles cuando se aprovecha el brokerage, cuando la organización tiene una curva de aprendizaje positiva que le da expertise al interior y cuando desarrolla capacidad de agencia. No obstante, a pesar de su importancia, ni el aprovechamiento del brokerage, la construcción de expertise o la capacidad de agencia son suficientes para explicar cómo la Unión se convirtió en generadora de procesos de desarrollo regional. La UCIRI pudo muy bien adquirir estas tres características pero ser sólo una organización fugaz que no lograra cohesión interna y que, por el contrario, generara incentivos económicos para que los pequeños productores buscaran mejorar sus ingresos individuales y nada más. Esto es particularmente relevante en momentos en que otras Осв en

\footnotetext{
${ }^{10}$ Asociación para el fomento de la cafeticultura sustentable, la producción orgánica en apoyo a organizaciones cafetaleras en México. Su sede está en San Cristóbal de las Casas, Chiapas.

${ }^{11}$ La CLAC coordina 300 organizaciones que agrupan a más de 200,000 productores latinoamericanos vinculados al $\mathrm{CJ}$.
} 
la región pierden miembros o se convierten en cascarones. ${ }^{12}$ La diferencia entre la UCIRI y otras organizaciones es que ésta no sólo se ha concentrado en sus logros hacia el exterior, sino que ha atendido también a sus procesos internos, generando una buena dosis de legitimidad entre sus socios. Esto lo analizamos en la siguiente sección.

\section{Principales mecanismos de gobierno en la UCIRI}

La otra dimensión que consideramos explica el éxito de la UCIRI es lo que sucede dentro del perímetro organizacional. Al interior, la Unión se ha esforzado por tener mecanismos de gobierno estables y aceptados, como la participación de su membrecía en estructuras de representación, toma de decisiones incluyente y transparente, así como liderazgos plurales y bien organizados. Todos estos elementos contribuyen en buena medida a explicar el éxito de la UCIRI hacia el interior.

\subsection{Membrecía}

La UCIRI es una organización abierta a cualquier pequeño productor de café de la zona, que desee afiliarse de manera individual. En la Unión no se excluye a nadie por su preferencia religiosa, de partido u origen étnico; con la excepción de acaparadores chicos y grandes, quienes pueden ingresar a la organización cuando "hagan público su deseo de no seguir con la maña de aprovecharse del trabajo de otros" (UCIRI, s/f).

La Unión no utiliza mecanismos de afiliación colectiva para servir a partidos políticos, confederaciones u otras agrupaciones de campesinos (VanderHoff, 2002; UCIRI, 2007), como sí hacen o han hecho numerosas organizaciones en el área.

\subsection{Representación}

La organización está gobernada por representantes comunitarios que se reúnen para integrar la Asamblea de Delegados, el máximo órgano de deliberación y decisión (Almanza, 2005). Los delegados toman las decisiones "acerca del rumbo que tomará la organización" (Jurado, 2005: 24), $\mathrm{y}$ asignan funciones y tareas a comisiones o representaciones.

Un punto fundamental en el esquema de representación es que todos los cargos, en los ámbitos general y comunitario, se deciden por votación directa, con propuestas desde las comunidades y a través de la conforma-

\footnotetext{
${ }^{12}$ Entrevista con Francisco Vanderhoff, asesor y socio de la UCIRI, 17 de septiembre de 2008, Crucero Buena Vista, Santiago Lachiguiri, Oaxaca.
} 
ción de planillas (Jurado, 2005; Mazariegos, 2006). Otro de los elementos más importantes en el esquema de representación de la UCIRI, que contrasta con las organizaciones de campesinos del periodo corporativo mexicano, es la constante rotación de los puestos de dirección y representación (VanderHoff, 2002). Los miembros del Consejo de Administración, ${ }^{13}$ del Consejo de Vigilancia y los delegados oficiales permanecen tres años en el cargo; mientras que los de las mesas directivas y los consejos de vigilancia, de cada comunidad, duran un año o dos. En todos los niveles de gobierno está prohibida la reelección y se puede poner en práctica la revocación de mandato, lo que materializa el supuesto del lugar vacío que toda democracia debe obedecer (Lefort, 1990).

\section{Organigrama UCIRI}

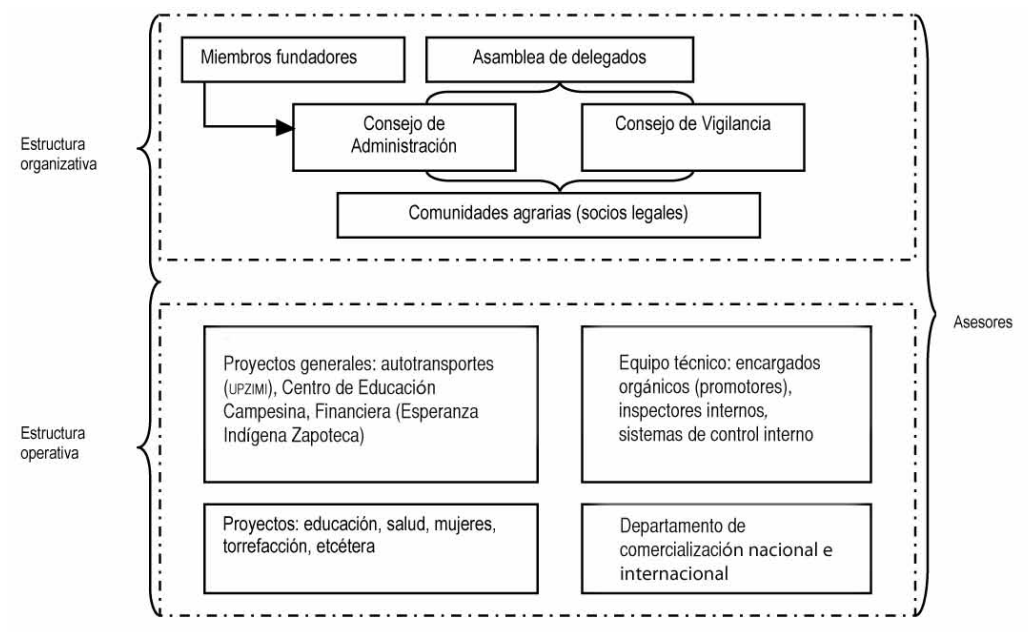

Fuente: Elaboración propia con información de Jurado (2005), Mazariegos (2006) y UCIRI (2007).

\subsection{Mecanismos de toma de decisiones}

Un aspecto fundamental en el tema de la toma de decisiones es que ninguna resolución sobre la conducción de la organización se toma por votación, por lo que la construcción de consensos es tarea cotidiana y permanente. Por ello, la deliberación y la negociación son fundamentales en la UCIRI. Éste no es un tema simple en una organización con numerosos socios, ubicados en diferentes comunidades dispersas en un territorio amplio y con serias limitaciones de transporte y comunicaciones (VanderHoff, 2005).

\footnotetext{
${ }^{13}$ Órgano de dirección y representación legal de la UCIRI.
} 
Otro asunto clave es el papel que han jugado los asesores, quienes están presentes en todos los procesos de toma de decisiones, con la misión de contribuir a enriquecer la discusión y presentar mapas completos de los asuntos que se discuten. En los órganos de representación no tienen derecho a voto pero sí a voz.

\subsection{Liderazgo complejo}

La UCIRI tiene una forma de liderazgo muy particular en varios sentidos.

Primero, un liderazgo tradicional acotado. Es lógico pensar que en una organización mayoritariamente indígena, las formas tradicionales de liderazgo serían muy significativas. En la UCIRI éstas no son ignoradas y forman parte del proceso de toma de decisiones. Sin embargo, hay candados y contrapesos que evitan que la hegemonía de los líderes tradicionales controlen a la organización.

Segundo, el liderazgo paralelo. Los asesores y miembros fundadores de la UCIRI nutren la toma de decisiones con un liderazgo paralelo que, aunque es menos formal, no es menos importante. Estos personajes participan en las deliberaciones con una racionalidad más instrumental, presentando escenarios posibles y deseables, no siempre contemplados por los miembros de las comunidades. Todo esto representa una fuente de información valiosa y una referencia particularmente útil.

Tercero. La rotación del liderazgo. La movilidad de los miembros en sus cargos permite que constantemente se ensanche la base de miembros capacitados en los mecanismos de funcionamiento y la toma de decisiones. Más aún, la existencia de un creciente número de ex líderes y ex dirigentes conocedores de los procesos internos, que permanecen como miembros activos, es un baluarte para la organización, debido a que participan en debates y discusiones sobre la producción, la comercialización o la orientación de proyectos productivos.

$\mathrm{Al}$ combinar estos liderazgos formales e informales y al rotarlos y renovarlos, la UCIRI no pierde expertise, sino que la multiplica, lo que posibilita una deliberación incluyente, plural e informada y fortalece la capacidad de los miembros para demandar la rendición de cuentas.

De lo anterior se puede ver que la Unión no sólo se preocupó por tender puentes hacia afuera, sino que paralelamente fue trabajando en procesos internos propios para ser una organización campesina autónoma, incluyente, plural y democrática. Así, la Unión, a pesar de sus fuertes vínculos con otros actores del comercio internacional, que en el aspecto de organización la pudieron haber acercado más hacia formas asociativas más comunes en el mercado, o que pudieron generar incentivos individuales para la corrupción o la fragmentación, se pudo mantener como 
un movimiento genuino de pequeños productores. Este éxito no es resultado de sus alianzas hacia afuera sino que es endógeno; es decir, está basado en la forma en que la organización ha cimentando diversos aspectos de su gobierno interno, como una membrecía incluyente, la representación y liderazgos complejos, así como una toma de decisiones transparente. La forma en que la UCIRI ha manejado estos elementos de gobierno le ha permitido construir: a) un sistema eficiente y efectivo de conducción que, al delegar, hace más eficiente su operación; b) mecanismos para la socialización de la comunicación e interacción entre los socios y para facilitar la deliberación y la construcción de consensos; c) formas de decisión que le dan legitimidad frente a sus miembros, comunidades y otros actores de su entorno, y d) procesos de rotación de roles y representación que acotan el liderazgo tradicional y generan horizontalidad, rotación y constante renovación de recursos humanos al interior de la organización.

Sin embargo, las alianzas con el exterior y la solidez interna no son suficientes para explicar el éxito de la UCIRI como detonadora de procesos de desarrollo regional. La Unión bien pudo convertirse en una cooperativa exitosa pero no trascender como actor de cambio social, como ha sucedido con otras cooperativas en la región (Natal, 2005). En cambio, al no ser la UCIRI una mera organización de campesinos sino una organización de comunidades, se ha constituido como una herramienta efectiva de integración comunitaria, capaz de promover acción colectiva para detonar procesos de desarrollo regional, aspecto que analizamos a continuación.

\section{La UCIRI: polo de desarrollo regional}

En los párrafos anteriores analizamos dos factores que nos ayudan a entender el éxito de la UCIRI: la construcción de alianzas con el exterior y un gobierno interno sólido. En esta sección mostramos cómo estos dos factores se han traducido sistemáticamente en proyectos para la región, lo que ha convertido a la UCIRI en un polo de desarrollo regional.

De acuerdo con las reglas del CJ, desde sus inicios la UCIRI ha destinado una parte de las ganancias de manera colectiva, para impulsar proyectos sociales no necesariamente relacionados con la producción del café. Así, a mediados de los ochenta fundó la empresa de transporte UpZIMI, s.C.L. ${ }^{14}$ que con tarifas más bajas que las de empresas de los coyotes bajó el costo del transporte en la región. ${ }^{15}$ En la misma época se formó el Centro de

\footnotetext{
${ }^{14}$ El transporte era uno de los problemas más agudos en la región. La Sierra Juárez de Oaxaca es una región montańosa de difícil acceso, prácticamente sin caminos ni transporte público.

${ }^{15}$ Estos proyectos afectaron intereses locales, lo que quizá detonó cierta violencia hacia la organización (amenazas, sabotajes y 37 socios asesinados) (VanderHoff, 2002; Vanderhoff y Roozen, 2002).
} 
Educación Campesina (CEC), proyecto de pequeñas clínicas de salud en poblaciones que carecían del servicio. En este mismo periodo se crearon pequeñas tiendas de abasto que surtían productos básicos a precios bajos. En los noventa la UCIRI crea los servicios comunitarios de radiocomunicación, instala una ferretería ${ }^{16}$ y estructura un fondo de ahorro y crédito.

En 1994 se terminó la construcción de la clínica de salud en Lachivizá, y en 1996 arrancó el proyecto para promover la igualdad de género al interior de la UCIRI. En la década de 2000 se abre una planta manufacturera de ropa que emplea a hijos de los productores. Un año después se formó un fideicomiso de garantía líquida para obtener créditos con el apoyo del Fideicomiso de Riesgo Compartido, una de las agencias del gobierno federal encargada de promover los agronegocios.

En 2004 la UCIRI es aceptada en el programa de Agencias de Desarrollo Local (ADL), y recibe fondos de la Secretaría de Desarrollo Social para proyectos de diversificación de la producción agrícola y renovación de los cafetales, entre otros. Un año después, con remanentes de la venta internacional de café, se inicia el proyecto de ecoturismo. En este mismo año se emprende uno de los proyectos más exitosos: Esperanza Indígena Zapoteca, la primera cooperativa de servicios financieros de la región, que permitió a los pobladores de la Sierra Juárez, por primera vez, acceso al crédito y al ahorro.

En el año 2005 la UCiri propuso a la Comisión Nacional de Áreas Naturales Protegidas (Conanp) la certificación y defensa coordinada de áreas naturales en la región, propuesta que pronto fue imitada por otras organizaciones de productores (Cobo y Bartra, 2007). A partir de 2006 la organización entró en un periodo de reestructuración en el que algunos de estos proyectos se han replanteado con el objetivo de hacerlos autosustentables, buscando que dejen de depender de la inyección continua de capital producto de las ventas del café, sin dejar de ser una herramienta de desarrollo para la región.

Con todo, también hay que ser claros en los alcances de la organización y el modelo de cJ. A partir de lo anterior, se podría pensar que el cJ, al pagar un precio justo y al usar el sobreprecio para atender necesidades sociales, va a tener efecto de forma rápida y sostenida en la vida de los productores y sus familias. Esto es inexacto. Si bien es cierto, como lo muestra el caso de la UCIRI, que algunos beneficios se manifiestan desde los primeros momentos, éstos no lo hacen en un volumen que signifique una transformación radical en el ingreso de los productores pobres. El grado y forma en que la membrecía modifica sus condiciones de vida está en función de una multiplicidad de factores, como educación, disposición,

\footnotetext{
${ }^{16}$ Que vendió productos para el trabajo agrícola a precios bajos.
} 
cercanía del recurso, ubicación territorial, cambios climatológicos, entre otros, cuya explicación excede los alcances de este trabajo y que hemos analizado en otros espacios (Natal 2001, 2004).

Lo que sí muestra el breve recorrido por tres décadas de la organización, que hicimos en los apartados anteriores, es que las alianzas hacia el exterior y la solidez interna se fueron traduciendo en proyectos que atendían la problemática local. Estos proyectos muestran: 1) la notable inversión económica y social que la Unión ha hecho en la región con las ganancias sociales del café, producto, en buena medida, del cJ; 2) que esta inversión ha resuelto necesidades apremiantes y problemas concretos de la región; y que estos proyectos enseñan además que 3) a través de ellos, la Unión ha provisto de servicios a miembros y no miembros, contribuyendo al desarrollo no sólo de su membrecía, sino de las comunidades de su región, lo que a su vez le ha ganado el reconocimiento de la población en general.

No obstante, estos avances en la atención de los problemas atingentes de la región, por sí mismos no serían suficientes para entender el éxito de la UCIRI como agente detonador de desarrollo. Para entenderlo hay que reconocer que la UCIRI ha sabido traducir sus alianzas con otros actores y su éxito comercial en acciones constantes de transformación de la realidad social de su territorio; y al mismo tiempo ha atendido aspectos de su gobierno interno, como una representación reconocida, participación amplia en la toma de decisiones y liderazgo complejo, elementos que le han permitido a los miembros lograr un mayor empoderamiento y experiencia asociativa, además de una base organizativa sólida y autónoma, que los independiza de la cooptación y los posibilita para sostener su propio desarrollo.

\section{A manera de conclusiones}

Este documento inició con la pregunta: ¿cómo una organización indígena de base puede jugar un papel tan significativo en el desarrollo regional? Para responderla intentamos explorar algunos de los factores exógenos y endógenos que la fortalecen; en particular señalamos tres que nos parecen centrales: a) la construcción de alianzas estratégicas con actores clave en el entorno; b) un gobierno interno sólido, democrático y plural, y c) una atención permanente a las necesidades de su territorio.

En varios sentidos, construir alianzas estratégicas con actores clave fue determinante para el éxito de la UCIRI. En parte porque la capacidad de la Unión para aprovechar el brokerage realizado por estos actores le permitió acceder a nichos de mercado y aprovechar oportunidades comerciales importantes. Más aún, la habilidad de la UCIRI para aprender de estos actores y construir sus propias capacidades le permitieron abrirse 
oportunidades de mercado, e incluso modificar arreglos institucionales que no le eran benéficos. Sin embargo, como hemos mostrado, la UCIRI también aprendió de estas alianzas y fue construyendo capacidades a su interior que le permitieron ganar independencia. Al mismo tiempo, la Unión no sólo promovió nuevos arreglos institucionales en el cJ, sino que en un ejercicio de solidaridad socializó saberes y experiencias asociativas con otras organizaciones pares. Esto no es menor, ya que tanto el asociacionismo es una precondición para iniciar el desarrollo (Easman y Uphoff, 1984), como la articulación del capital social para su sustentabilidad (Putnam, 1993).

La UCIRI logró también construir un gobierno interno con horizontalidad en el proceso de toma de decisiones, representación y candados de liderazgo, que dio confianza a sus miembros y les permitió apropiarse de procesos y estructuras de gobierno. Esto no sólo generó legitimidad y una organización sólida; sino también aprendizajes cívicos de fundamental importancia para sus miembros. Entre estos destacan: 1) la preparación de la membrecía para el ejercicio de la ciudadanía al hacerlos autoconscientes de sus derechos y obligaciones; 2) un ejercicio de tolerancia en la deliberación y empoderamiento en el uso de la voz (Hirschman, 1977). Asimismo, la movilidad del liderazgo obligó a los miembros a participar y redujo las asimetrías de información al interior; pero además 3) generó relaciones más horizontales en la organización, sin romper radicalmente con los liderazgos tradicionales y, finalmente, 4) el hecho de que algunos miembros participen como representantes de su comunidad y que sus puestos sean temporales, rompe el secretismo, socializa la información y, por tanto, se genera apropiación. Más aún, esto contribuye a romper con las relaciones verticales y de autoridad y genera aprendizajes en transparencia y rendición cuentas. Todo esto crea confianza y lazos comunitarios más sólidos que fortalecen la cooperación. De tal suerte que la participación en la UCIRI se convierte en una experiencia asociativa y democrática, que empodera a los individuos para ejercer su voz y demandar rendición de cuentas, incluso allende su organización.

La capacidad de la Unión para traducir sus vínculos con el exterior y gobierno sólido en la creación de bienes comunes se explica en buena medida porque la UCIRI es una organización de base, una iniciativa local desde abajo. Esto le ha permitido cohesionar a un grupo amplio de personas y sus comunidades facilitando la apropiación, elemento clave para el éxito organizacional y de cualquier proyecto de desarrollo. Sin embargo, no se puede concluir a cabalidad si fue su éxito en el mercado global o si fue su sólido gobierno interno o su atención a problemas sociales sentidos, lo que dio confianza de propios y extraños en la organización y la convirtió en polo de desarrollo regional. Lo más probable es que haya 
sido la concatenación y retroalimentación de estos elementos lo que generó un círculo virtuoso para el desarrollo.

Lo que resulta evidente es que en este círculo virtuoso se resolvió uno de los principales problemas del desarrollo participativo: la reconciliación de intereses individuales y colectivos. En el caso de la Unión, los intereses individuales han estado atendidos vía los ingresos por productor resultado de la venta del café a través del CJ e innovadores instrumentos de crédito; de tal suerte que los socios de la UCIRI pueden maximizar sus intereses individuales y generar beneficios para ellos y sus familias. Pero por el otro lado, el hecho de que en los esquemas del cJ la ganancia social debe ser invertida para resolver problemas locales, hace que las осв involucradas desarrollen un espiritu público, es decir, que compartan los beneficios privados invirtiendo en causas sociales. Esto, aunado al interés de la Unión por influir en su entorno, ha hecho que ésta inicie proyectos para atender las necesidades más apremiantes de las comunidades de la región, beneficiando así a miembros y no miembros. Esto no sólo le ha ganado legitimidad, sino que también ha fortalecido en sus miembros una cultura de interés por los asuntos sociales relevantes para la región, o en términos de Walzer $(1998,2010)$, ha reforzado la virtud civica de los socios. Al mismo tiempo, el interés de la organización por la creación de bienes públicos (la escuela, la clínica de salud, etc.) ha hecho que sus miembros puedan transitar de lo que Banfield (1958) llama amoral familism, la maximización de los intereses del núcleo familiar, a un ethos más orientado a lo público, a lo colectivo. Esto les ha permitido a los socios de la UCIRI trascender la dicotomía clásica entre egocentrismo y altruismo, lo que facilita la acción colectiva. Éste es un ejemplo notable de reconciliación de intereses en el que el comercio se vuelve la arena para el desarrollo colectivo más que el campo de batalla de intereses individuales en conflicto.

Finalmente, el caso de la UCIRI nos lleva a reflexionar sobre la actual conceptualización del desarrollo regional y la necesidad de repensar el papel de las organizaciones de base en este proceso. Este estudio intentó mostrar las oportunidades que existen al impulsar y fortalecer a las organizaciones de grupos excluidos de los procesos de globalización económica. Asimismo, este caso indica que puede haber oportunidades en las políticas públicas para fortalecer a las осв regionales, y a través de ellas iniciar un proceso de desarrollo desde abajo, como hemos discutido en otros espacios (Natal, en prensa). Ejemplos como el de la Unión nos permiten continuar sońando y seguir creyendo en horizontes prometedores de participación e inserción de los pobres del campo en procesos sostenibles de desarrollo regional. 


\section{Agradecimientos}

Queremos mostrar nuestro agradecimiento a los comentarios que hicieron los dictaminadores anónimos al presente trabajo. De igual manera, agradecemos a los miembros del Seminario Permanente Institucional del Instituto de Investigaciones Sociales de la UNAM sobre "Las organizaciones campesinas frente al Estado y la política”, por sus sugerentes recomendaciones y cuestionamientos; en particular a Hubert Carton De Grammont, su director, así como a Bruno Lutz.

\section{Bibliografía}

Almanza, Horacio (2005), "La Unión de Comunidades Indígenas de la Región del Istmo (UCIRI)”, Vinculando.org, <http://vinculando. org/comerciojusto/mst_comercio_justo/uciri.html>, diciembre de 2009.

Aranda, Josefina y Carmen Morales (2002), "Poverty Alleviation through Participation in Fair Trade Coffee: The Case of cepco", Universidad de Colorado, <http://www.colostate.edu/Depts/Sociology/ FairTradeResearchGroup, noviembre de 2009.

Aranda, Josefina (2003), "Peasant Farmers in the Global Economy: The State Coalition of Coffee Producers of Oaxaca", en Timothy Wise, Hilda Salazar y Laura Carlsen (eds.), Confronting Globalization: Economic Integration and Popular Resistance in Mexico, Kumarian Press, Bloomfield, pp. 149-170.

Arditi, Benjamín y Carlos Chávez (2006), "Del comercio libre al comercio justo. Los nuevos defensores de la igualdad en las relaciones Norte-Sur", Revista Sistema, 195, Fundación Sistema, Madrid, pp. 53-82.

Banfield, Edward (1958), The Moral Basis of a Backward Society, Free Press, Nueva York.

Bauman, Zygmunt (2001), La globalización, consecuencias humanas, Fondo de Cultura Económica, México.

Cobo, Rosario y Armando Bartra (2007), Puerta del Viento. Cerro de las Flores, Área comunitaria protegida, Instituto Maya, México. 
Durnez, David (2004), "Does Fair Trade encourage sustainable development? Could it be considered as a development project? Impact analysis of Fair Trade practices in the export of craft products from the region of Kolkata", Mémoire en vue de l'obtention du diplôme d'études spécialisées (DES) en Gestion du Développement, promotor G. Pirotte, Université de Liège, Lieja.

Easman, Milton y Nornam Uphoff (1984), Local Organizations: Intermediaries in Rural Development, Cornell University Press, Ithaca.

Fama, Eugene y Michael Jensen (1963), "Separation of ownership and control”, Journal of Law and Economics, 26, University of Chicago Press, Chicago, pp. 301-326.

Fama, Eugene (1980), "Agency Problem and the Theory of the Firm", Journal of Political Economy, 88, University of Chicago Press, Chicago, pp. 388-407.

FLO (Fairtrade Labelling Organisations) (2010), "Annual Report 20092010. Growing Stronger Together”, <http://www.fairtrade.net/ fileadmin/user_upload/content/2009/resources/FLO_AnnualReport-2009_komplett_double_web.pdf>, agosto de 2010.

Flores-Olea, Víctor y Abelardo Mariña (1999), Critica de la globalidad. Dominación y liberación en nuestro tiempo, Fondo de Cultura Económica, México.

Fridell, Gavin (2006), "Fair Trade and Neoliberalism. Assesing Emerging perpectives", Latin American Perspectives, 151, 33 (6), Sage, Thousand Oaks, California, pp. 8-28.

Fridell, Gavin (2007), Fair Trade Coffee. The prospects and Pitfalls of MarketDriven Social Justice, University of Toronto Press, Toronto.

Hirschman, Albert (1977), Salida, voz y lealtad: respuestas al deterioro de empresas, organizaciones y Estados, Fondo de Cultura Económica, México.

Ianni, Octavio (1996), Teorías de la globalización, Siglo XXI-Universidad Nacional Autónoma de México, México. 
Jaffee, Daniel (2007), Brewing Justice. Fair Trade Coffee, Sustainability, and Survival, University of California Press, Los Ángeles.

Jolette, Helene (2001), The Fair Trade Marketing of Handloom Products from South India, École des Hautes Études Commerciales, Montreal.

Jurado-Celis, Silvia Nuria (2005), “Educación popular para el desarrollo rural en comunidades cafeticultoras del Istmo de Tehuantepec en Oaxaca", tesis para obtener el título de ingeniera agrícola, Universidad Nacional Autónoma de México, edición del autor.

Lefort, Claude (1990), La invención democrática, Nueva Visión, Buenos Aires.

Mazariegos-Sánchez, Adriana (2006), "La Unión de Comunidades Indígenas de la Región del Istmo (UCIRI), Oaxaca: estrategia y gestión de la certificación de los procesos de producción de café", borrador de tesis doctoral, Universidad Autónoma Metropolitana, Iztapalapa, México.

Natal, Alejandro (2001), The Political Economy of Community Self-help Development: The Costs of Governing Participation, El Colegio Mexiquense, Zinacantepec.

Natal, Alejandro (2004), Information Costs and Local Institutions: Participatory Decision Making in a Rural Community, El Colegio Mexiquense, Zinacantepec.

Natal, Alejandro (2005), Women Organisations, Social Change and Social Capital: Mazunte, El Colegio Mexiquense, Zinacantepec.

Natal, Alejandro (en prensa), "Agencias de desarrollo local: capital social y vinculación social”, El Colegio Mexiquense-Indesol, Zinacantepec.

Oxfam International (2002), Mugged: Poverty in your Coffee Mug, <http:// www.maketradefair.com/assets/english/mugged.pdf $>$, marzo de 2006. 
Pariente, William (2000), The impact of fair trade on a coffee cooperative in Costa Rica. A producers behaviour approach, Université Paris I Panthéon Sorbonne, París.

PNUD (Programa de las Naciones Unidas para el Desarrollo) (2004), Indicadores municipales de desarrollo humano en México, PNUD, México, <http://www.undp.org.mx/desarrollohumano/disco/ index.html>, enero de 2010.

Putnam, Robert (1993), Para hacer que la democracia funcione: la experiencia italiana en descentralización administrativa, Galac, Caracas.

Renard, Marie-Christine (1999), Los intersticios de la globalización. Un label (Max Havelaar) para los pequeños productores de café, CEMCACePCo-ISMAM, México.

Renard, Marie-Christine (2005), "Quality Certification, Regulation and Power in Fair Trade", Journal of Rural Studies, 21 (4), Elsevier Science, Amsterdam, pp. 419-431.

Reynolds, Laura, Douglas Murray y Peter Leigh Taylor (2003), One Cup at a Time: Poverty Alleviation and Fair Trade in Latin America, Centro de Investigaciones y Estudios Superiores en Antropología Social-Colorado State University-Desarrollo Alternativo, A.C., $<$ http:/www.colostate.edu/Depts/Sociology/FairTradeResearchGroup/doc/fairtrade.pdf\#search='one $\% 20$ cup $\% 20$ at $\% 20 \mathrm{a} \% 20$ time\%20raynolds>, enero de 2006.

Ronchi, Loraine (2002), "The Impact of Fair Trade on Producers and Their Organizations: A case Study with Coocafé in Costa Rica”, prus Working Paper No. 11, <http://www.fairtrade.net/uploads/media/ ronchi_ft_impact_cococafe_costa_rica.pdf>, febrero de 2008 .

Rubio, Blanca (1996), Explotados y excluidos. Los campesinos latinoamericanos en la fase agroexportadora neoliberal, Plaza y Valdés, México.

Simpson, Charles y Anita Rapone (2000), "Community Development From the Ground Up: Social-Justice Coffee", Human Ecology Review, 7 (1), Society for Human Ecology, Bar Harbor, Maine, pp. 46-57, <http://www.humanecologyreview.org/pastissues/ her71/71simpsonrapone.pdf>, diciembre de 2007. 
Solidaridad (2006), http://www.solidaridad.nl/indexspanje.html, marzo de 2006.

uciri (Unión de Comunidades Indígenas de la Región del Istmo) (2007), “QQuiénes somos?”, <http://www.uciri.org/espanol/espanol.htm>, enero de 2007.

uciri (Unión de Comunidades Indígenas de la Región del Istmo) (s/f), Una autopresentación, http://www.demexicoalmundo.com.mx/ exporganicos/Ponencias/ExportacionOrganicosUCIRI.doc, diciembre de 2007.

Unión Majomut (2008), “Introducción”, <http://www.majomut.org/ introduccion/introduccion.html>, mayo de 2008.

Utting-Chamorro, Karla (2005), "Does fair trade make a difference. The case of small coffee producers in Nicaragua", Development in Practice, 15 (3-4), Oxfam GB, Oxford, pp. 584-599.

VanderHoff, Frans (2005), Excluidos hoy, protagonistas mañana, UCIRI, México.

VanderHoff-Boersma, Frans y Nico Roozen (2002), La aventura del comercio justo, una alternativa de globalización, por los fundadores de Max Havelaar, El Atajo, México.

VanderHoff- Boersma, Frans (2002), "Poverty Alleviation through Participation in Fair Trade Coffee Networks: The Case of uciri, Oaxaca, Mexico", <http://www.colostate.edu/Depts/Sociology/ FairTradeResearchGroup/doc/pov.pdf>, diciembre de 2007.

Walzer, Michel (1998), Tratado sobre la tolerancia, Paidós, Barcelona.

Walzer, Michel (2010), Pensar políticamente, Paidós, Barcelona.

Recibido: 4 de febrero de 2011.

Reenviado: 3 de agosto de 2011. Aceptado: 19 de septiembre de 2011. 
Carlos Chávez-Becker. Es maestro en estudios políticos y sociales por la Universidad Nacional Autónoma de México (UnAm). Su trabajo de investigación se ha centrado en diversas formas y alternativas de acción colectiva en México: organizaciones de la sociedad civil y movimientos sociales. Sus líneas de investigación son movimientos sociales, sociedad civil, acción colectiva y procesos de democratización en América Latina. Entre sus publicaciones recientes se encuentran: en coautoría, "Fair Trade in Mexico: New opportunities for local and political development", en Vibeke Andersson y Steen Fryba Christensen (eds.), New Latin American Development Strategies in a Changing International Economic and Political Context. Development Research Series, Occasional Papers, 7, Aalborg University, Aalborg, Dinamarca, pp. 74-98 (2008); en coautoría, "Del comercio libre al comercio justo. Los nuevos defensores de la igualdad en las relaciones Norte-Sur", Revista Sistema, 195, Fundación Sistema, Madrid, pp. 53-82 (2006); "Relevancia social y gobernanza democrática: una aproximación desde el desempeño organizacional”, en Cristina Puga y Matilde Luna (coords.), Nuevas perspectivas para el estudio de las asociaciones, Anthropos-unam, México, pp. 205-232 (2010).

Alejandro Natal. Es doctor en estudios del desarrollo por la London School of Economics, Reino Unido. Actualmente es profesor-investigador en El Colegio Mexiquense y coordina el Programa Interdisciplinario de Estudios del Tercer Sector. Dirige el Observatorio de la Sociedad Civil y es miembro del Consejo Editorial del Journal of Civil Society, de la Universidad de California, en Los Ángeles, y de la Enciclopedia de la Sociedad Civil de la Universidad de Heidelberg. Es miembro del Sistema Nacional de Investigadores, nivel I. Sus líneas de investigación son: sociedad civil, capital social y desarrollo comunitario. Entre sus últimas publicaciones destacan: "La sociedad civil como espacio de conflicto: el caso de Valle de Bravo", en Pablo Castro y Héctor Tejera (coords.), Participación y ciudadanía en México, Universidad Autónoma Metropolitana, IztapalapaJuan Pablos, México, pp. 229-263 (2010); “El entorno organizacional de las osc: una aproximación a su estudio", en Matilde Luna y Cristina Puga (coords.), Nuevas perspectivas para el estudio de las asociaciones, Antrophos, Barcelona, pp. 192-204 (2010); "Corrupción y transparencia. El permanente dilema en el Estado y la sociedad. Efectos y consecuencias sobre el sistema político y la construcción democrática”, en Leticia Salomón (coord.), Democracia y gobernabilidad: evaluación y perspectivas, CEDOH-ASDIUNAH, Tegucigalpa, pp. 107-123 (2010); "La sociedad civil mexicana como actor de cambio social. Un recuento de su contribución a la transición democrática”, en Henio Millán, Marta Gloria Morales y Luis Alberto Fernández (coords.), Cambio político y déficit democrático en México, 
Miguel Ángel Porrúa-Universidad Autónoma de Querétaro, México, pp. 167-187 (2010); en coautoría, "Civil Society and Social Capital in Mexico and Central America”, en Stefan Toepler y Regina List (coords.), International Encyclopedia of Civil Society Helmut Anheier, Springer, Nueva York, pp. 262-268 (2010). 